\title{
BMJ Open Quality Taming the chaos: NHS professionals' perspective of using video consulting during COVID-19 in Wales
}

Gemma Johns (D) , ${ }^{1}$ Sara Khalil, ${ }^{1}$ Mike Ogonovsky, ${ }^{1}$ Markus Hesseling, ${ }^{2}$ Allan Wardhaugh, ${ }^{3}$ Kerrie Phipps, ${ }^{4}$ Jessica Williams, ${ }^{1}$ Bethan Whistance, ${ }^{1}$ Alka Ahuja ${ }^{1}$

To cite: Johns G, Khalil S, Ogonovsky M, et al. Taming the chaos: NHS professionals' perspective of using video consulting during COVID-19 in Wales. BMJ Open Quality 2021;10:e001318. doi:10.1136/ bmjoq-2020-001318

- Additional supplemental material is published online only. To view, please visit the journal online (http://dx.doi.org/10. 1136/bmjoq-2020-001318).

Received 18 December 2020 Accepted 10 December 2021

Check for updates

(c) Author(s) (or their employer(s)) 2021. Re-use permitted under CC BY-NC. No commercial re-use. See rights and permissions. Published by BMJ.

${ }^{1}$ Technology Enabled Care Cymru, Aneurin Bevan University Health Board, Gwent, UK ${ }^{2}$ Betsi Cadwaladr University Health Board, Bangor, UK

${ }^{3}$ Cardiff and Vale UHB, Cardiff, UK

${ }^{4}$ Hywel Dda Health Board, Carmarthen, UK

Correspondence to

Gemma Johns;

gemma.johns3@wales.nhs.uk

\section{ABSTRACT}

The use of video consulting (VC) in the UK has expanded rapidly during the COVID-19 pandemic. Technology Enabled Care (TEC) Cymru, the Welsh Government and Local Health boards began implementing the National Health Service (NHS) Wales VC Service in March 2020. This has been robustly evaluated on a large-scale All-Wales basis, across a wide range of NHS Wales specialities. Aims To understand the early use of VC in Wales from the perspective of NHS professionals using it. NHS professionals were approached by TEC Cymru to provide early data.

Methods Using an observational study design with descriptive methods including a cross-sectional survey, TEC Cymru captured data on the use, benefits and challenges of VC from NHS professionals in Wales during August and September 2020. This evidence is based on the rapid adoption of VC in Wales, which mirrors that of other nations.

Results A total of 1256 NHS professionals shared their VC experience. Overall, responses were positive, and professionals expressed optimistic views regarding the use and benefit of VC, even when faced with challenges on occasions.

Conclusions This study provides evidence of general positivity, acceptance and the success of the VC service in Wales. Future research studies will now be able to explore and evaluate the implementation methods used within this study, and investigate their effectiveness in being able to achieve better outcomes through VC.

\section{BACKGROUND}

Video consulting (VC) has increased through healthcare and social care delivery since the start of the COVID-19 pandemic. ${ }^{1-3}$ During 2020, VC use has expanded across the UK and throughout the National Health Service (NHS). The utilisation of VC, to this extent, is a new and innovative method of healthcare practice in the UK, in that its use and acceptance present a very different picture today than it did pre-COVID. ${ }^{34}$ There is emerging evidence that VC can deliver safe and timely care in many settings and offer several advantages to patients, families, professionals and society. ${ }^{5}$ Pilot studies do make up the majority of VC literature with small and often highly selected samples, ultimately questioning its use, benefits and challenges across services. ${ }^{7}$ Due to these issues with pilot studies, it means that there are clear gaps existing in the current evidence-base that do require urgent attention, ${ }^{8}$ and therefore the suggestion and need to continuously evaluate and re-evaluate on a national level will allow for sustainable VC platforms to be embedded for the long-term into healthcare and social care systems. ${ }^{9}$ Digital healthcare implementations across the UK can be challenging and have featured in a number of reports in recent years. ${ }^{10-13}$ To be able to improve implementations such as these, a series of recommendations have been adopted in Wales by Technology Enabled Care (TEC) Cymru ${ }^{14}$ and Local Health boards. ${ }^{15} 16$ Due to the collaboration between TEC Cymru, the Welsh Government, NHS Informatics service and various clinical teams employing this method of using data which are clinically driven and informed, it has ensured a large-scale nationwide implementation at a pace not achieved before. $^{17-20}$

\section{AIMS AND METHODOLOGY}

The aim of this study was to explore the professional perspectives of $\mathrm{VC}$ and its use, benefits and challenges.

The TEC Cymru Programme enables the sustainable use, scale-up and spread of digital services. As a response to the pandemic emergency, TEC Cymru implemented a new NHS Wales VC Service ${ }^{21}$ rolling out a single platform 'Attend Anywhere' to all NHS services. Since March 2020, TEC Cymru has rolled out and evaluated on a large-scale basis across a wide range of specialities across all Health Boards in Wales. This study is one part of the larger evaluation (with more than 50000 participants). This study used an observational study design with descriptive qualitative 
methods, including across-sectional survey that included a mixture of questions with matrix rating scales and freetext response questions capturing professional perspectives of $\mathrm{VC}$ and its use, benefits and challenges. By using a cross-sectional survey, it allowed for VC to be looked at on a range of dependent variables and to delve into what trends are occurring within the quantitative data. For challenges to VC, professionals were asked to rate the relevancy of the challenges and how difficult this would make VC for them and for their patients on a 4-point Likert scale, ranging from 1 (not at all relevant) to 4 (very relevant).

Professionals were also asked to complete 16 statements regarding their use of $\mathrm{VC}$, whereby they were to state whether they were 'true', 'false' or 'unable to say.

All NHS professionals (including clinical and administrative staff and managers) who were using VC in Wales were invited to participate, and a SurveyMonkey link was sent to them. Only a handful of professionals had used VC prior to COVID-19. The use of questionnaires is an underused but valid qualitative research tool. This format allowed for data collection at a time when face-to-face contact was prohibited and ensured a high degree of anonymity for participants. This allowed for wider participation of professional staff within NHS Wales, giving voice to a range of perspectives that may have been lost in online focus groups or online interviews, where existing relationships and power structures may have influenced people's responses. Although the questionnaire was limited to professionals within Wales only, it was open to all VC platform users (not just Attend Anywhere users). The questionnaire ran for 1 month between August and September 2020. A copy of the questionnaire is included as an online supplemental file.

TEC Cymru uses a Quality Improvement methodology to evaluate NHS Wales services. Data were analysed using reflexive thematic analysis. For the analysis to be reflexive in its nature, the data comments were coded to reflect researchers' interpretation of the data set and any theoretical assumptions associated with the analysis and resources of the researchers. This was a collaborative approach between TEC Cymru's researchers which led to defined topics. Themes were then developed through reflexive engagement with the data as above, and TEC Cymru obtained full ethical approval and risk assessments from Aneurin Bevan University Health Board Research \& Development Department (R\&D Reference Number: $\mathrm{SA} / 1114 / 20)$.

In Wales, there are seven Local Health Boards, and the care sectors are split into three categories, including Primary, Secondary and Community Care. See online supplemental appendices 1 and 2 for the breakdown of these categories. This article presents the All-Wales overview data from the questionnaire including 6 out of 12 questions ( 3 of these questions have additional subquestions, for example, matrix rating scales of 16 statements, 13 benefits and 8 challenges) with open-ended text boxes. For more in-depth and inclusive data sets, please see the TEC Cymru website. ${ }^{14}$ The exclusion of some of the questions was due to not being relevant to the article, for example, Health Board name, VC platform name, specialty (added in online supplemental appendix) and other comments. The full report including all questions can be found on TEC Cymru's website, ${ }^{22}$ alongside other data sets captured by TEC Cymru. ${ }^{23}$

There was no patient or public involvement in the development of this study. This was a professional only study.

\section{RESULTS}

For this study, there were a total of 1256 responses by professionals from seven Health Boards in Wales (at that point, 6787 users in NHS Wales had access to Attend Anywhere) The majority of responses were from Secondary Care $(\mathrm{n}=873)$ and Primary and Community Care $(n=217)$, and $n=37$ responses were from Management and Administration across a mix of care sectors. The respondent's demographics are detailed in table 1 .

\section{The use and value of VC}

All professionals were asked, 'Do you think that VC should be used for healthcare and social care appointments? (If it is clinically appropriate)'. In total, $79 \%$ responded 'yes', 20\% responded 'maybe' and 1\% responded 'no'. This distribution is shown in figure 1 .

Generally, professionals across Health Boards and care sectors had a positive regard towards the use of $\mathrm{VC}$ to deliver healthcare and social care. There were a wide range of reasons to support their preferences. These are divided into the following subcategories and displayed as narrative quotes.

\section{Flexibility}

Improved flexibility was observed as a factor as to why many professionals perceived $\mathrm{VC}$ to be of better use and value for managing patient care, compared with face-toface:

Working professionals not having to leave work to attend appointments. (Doctor, Primary care)

Appointment times can be more flexible to suit the patient's needs. (Psychiatry Nurse, Secondary care)

Offers the patient and health system flexibility. (Respiratory Medicine Nurse, Secondary care)

\section{Clinical suitability}

Some professionals reported that not all clinical conditions are suitable for VC; however, many state that VC is clinically suitable to reduce patient stress and anxiety levels, which in turn produces positive patient feedback:

Feedback I have had so far has been positive with patients who have reported feeling anxious to start with, but the overall experience was positive, and they chose to use it again ... (Rehabilitation Psychologist, Secondary care) 
Table 1 Respondent demographics

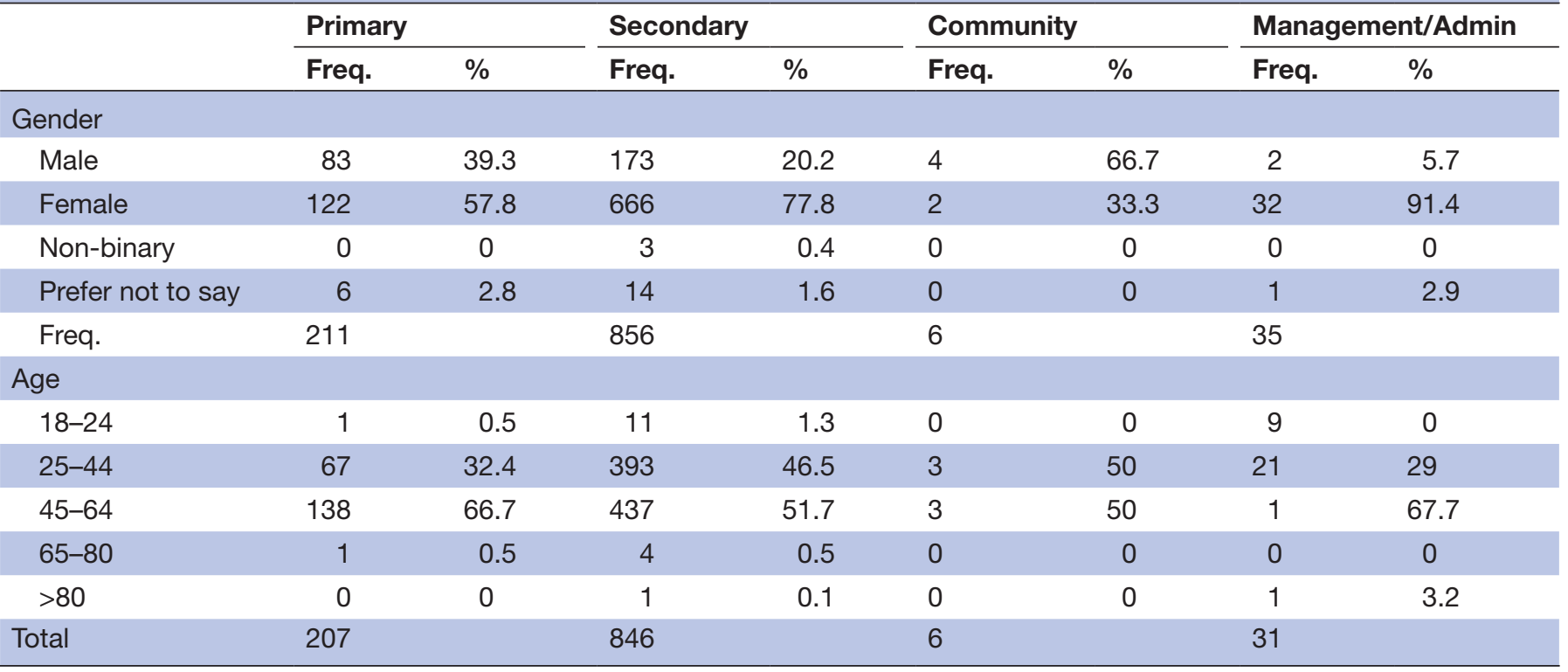

Patients have reported they often feel stressed when coming into hospital ... and since we have been using digital platforms, they feel much more able to attend and engage ... (Pain Management Nurse, Secondary care)

\section{Observing patients in their own environment}

The professionals' capability to be able to observe patients within their own environments was considered to deliver better care in some cases, particularly for younger patients:

Patients are more relaxed in their home environment.

(Speech and Language Therapist)

Enables patients to stay home and feel comfortable ... (Dietician, Secondary care)

Young persons are very familiar with using video calls anyway. They can be more open in this environment if familiar. (Psychologist, Secondary care)

\section{Independent care of younger patients}

Professionals report other advantages for younger patients, such as improved accessibility and independent care. When young people attend traditional face-to-face appointments, they often depend on their carers/family

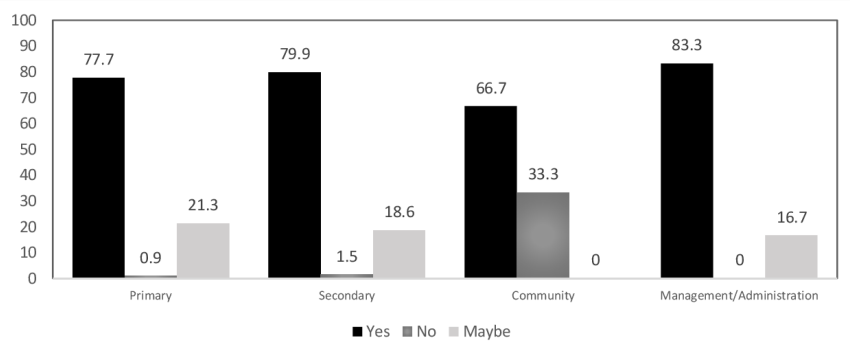

Figure 1 Distributions of responses to 'should VC be used in each care sector'. VC, video consulting. members to transport them to the clinic and they can often be passive recipients of care in such face-to-face settings:

Being able to provide VC means that young people can easily access the service. (Psychologist, Secondary care)

Lots of young people have been more able to attend appointments independently which they perceive as being a positive outcome. (Psychiatric Nurse, Secondary care)

\section{Clinical reservations and uncertainty}

The questionnaire demonstrates that $20 \%$ of professionals believe that VC should 'maybe' be used where possible. Professionals here state the following:

VC should be used in conjunction with face-to-face. (Psychiatric Nurse, Secondary care)

... A one-off appointment or quarterly face-to-face if care is ongoing would be ideal too. (Psychologist, Secondary care)

[VC] should continue to be used forever, however ... it shouldn't replace face-to-face. We should use VC as an option ... (Support Worker, Primary Care)

\section{True and false statements}

Overall, there were notable differences between Primary and Secondary Care on the responses, in that Secondary Care responded 'true' on more occasions than Primary Care. Considering some examples, it appears that Secondary Care 'regularly use VC' more than Primary Care, 'colleagues are keener to use VC' and their 'systems and bookings are adequately set-up' to allow for VC. However, Primary Care were more positive in their responses for statements, which were receiving 'adequate support and equipment', having 'sufficient internet connectivity' and 
less 'concerns about cyber security'. In Management and Administration, the majority of responses were 'true', except for the 'choice' of appointment, 'VC as equivalent to face-to-face' and that 'adequate support and equipment' are available. This may reflect the optimism and investment managers were willing to put into $\mathrm{VC}$ as a solution to tackle their ever-building waiting list and postCOVID-19 backlog.

\section{The benefits of VC}

Professionals were provided with 13 possible benefits to VC, Generally, Secondary Care were more positive in their responses than Primary Care, with a higher frequency of 'very beneficial' responses for every aspect, except for 'reducing the likelihood of Did Not Attends' (DNAs) (where Primary Care rated this as 'very beneficial'), although Primary and Secondary Care were similar in the responses they gave for the 'reduction of wait times'. Secondary Care were slightly more positive. The biggest difference between the two care sectors was in the evaluation of 'lowering rates of infection', in that $39.4 \%$ of Primary Care rated this as 'very beneficial' compared with $60.1 \%$ of Secondary Care. Overall, the responses were positive. For Management/Administration, 'saving time taken for travelling and parking' for patients and 'lowering the rates of infection' were rated highly. The lowest rated aspect was 'improvement of family involvement/support. Generally, professionals across Health Boards and care sectors had a positive regard towards the benefits of VC to deliver healthcare and social care. There was a wide range of reasons to support their preferences. These are divided into five subcategories and displayed as narrative quotes.

\section{Time and travel savings}

A large proportion of professionals reported that there had been significant savings in time and travel for both professionals and patients; nevertheless, more emphasis was placed on the benefits this had for their patients:

Saves travel time, especially for families with other children, saves professionals travel time. (Occupational Therapist, Secondary care)

Reduces the need for patients to travel long distances to appointments. (Management, Primary Care)

Travel and time savings were also noted as an additional benefit for patients with mobility and psychological and neurological difficulties:

Those with social anxiety, mobility problems able to access our service for the first time. (Psychiatry Administrator, Secondary care)

Allows for patients with mobility issues and/or limited access to transportation to attend with ease. (Psychologist, Secondary care)

I have clients who prefer virtual appointments and like the convenience of this. (Psychiatry Nurse, Secondary care)

\section{Environmental benefits}

There were also benefits expressed regarding the environment in a move away from face-to-face consultations:

I advocate for environmental issues and agree that for various reasons (paper waste, petrol, etc.) that video calls are more environmentally friendly than face-to-face ... big thumbs up! (Trainee Psychologist, Secondary care)

There is less impact on the environment, with less travel. (Palliative Medicine Doctor, Secondary care)

\section{Work/life balance and joined-up working}

Professionals identified a range of benefits that they considered specific to themselves when using VC, including an improved work/life balance, enhanced multidisciplinary team meetings (MDTs) and increased performance and flexibility of consultations:

Improved work-life balance- able to work from home more as a result ... can better time appointments. (Paediatrics Pharmacist, Secondary care)

More efficient and direct referrals within the MDT virtual clinic. (Genitourinary Medicine Doctor, Secondary care)

Enables teams from across the Health Board to communicate better, more frequently, with more of the team present (virtually). (Dietician, Secondary care)

In addition, joined-up working also highlighted the possibility of improved interactions for both professionals and patients with wider family involvement:

Does provide the opportunity for a second parent who may be working to be present and improve communication with both. (Physiotherapist, Secondary care)

Beneficial if it can involve family who would not normally be available in person. (Speech and Language Therapist, Secondary care)

\section{Lowered DNAs and increasing accessibility to services}

There were also other benefits mentioned, such as that of improved access to services, lowered rates of DNAs and the freeing up of telephone lines:

Allows more free telephone lines when booking appointments. (General practitioner (GP) Doctor, Primary care)

I have observed lower rates of DNAs when conducting video calls vs face-to-face appointments. (Trainee Psychologist, Secondary care)

They (patients) love the ease of it and I have had no DNAs since this has been used. (Mental Health Psychotherapist, Secondary care)

\section{Infection control}

The most prominent benefit reported within the data was the impact of the COVID-19 pandemic on the delivery 
of care; professionals expressed their gratitude for $\mathrm{VC}$ during this time:

Prevent[s] footfall into the surgery during the pandemic. (GP, Primary care)

With the current restrictions, there is definitely a place for VC in certain circumstances. (Rheumatology Doctor, Secondary care)

As a staff member who has had to shield and work from home, VC has enabled me to continue to support and provide intervention to my patients. (OT Cardiology, Secondary care)

Also allows for review of patients receiving palliative care without increasing the risk of COVID ... (GP, Primary care)

\section{Challenges of VC}

Generally, Primary and Secondary Care are similar in their ratings of potential challenges for professionals and patients using VC. One challenge that demonstrated a large difference was patients' 'access to a device', with Primary Care rating this as 'very relevant', compared with Secondary Care. Both care sectors demonstrated these challenges to be more relevant for their patients, than their own, which included 'access to a device', 'access to internet' and 'having a lack of confidence to use VC'. The challenge regarding a preference for face-to-face was rated similarly for both Primary and Secondary Care for themselves and their patients, but again large differences exist between what they consider more challenging for their patients, compared with their own challenges. For Community Care, all challenges were rated as 'not very relevant', and the least relevant was having 'a lack of confidence'. In terms of challenges for patients, 'access to a device' was also considered most relevant. Professionals in Community Care found their own preference for faceto-face or telephone as not relevant as they felt it was for their patients, where $83.3 \%$ of professionals stated this to be 'relevant'. In Management and Administration, it was rated that patients' difficulties were more relevant than professionals', which included 'poor internet connectivity' as the largest difference. On the contrary, the relevancy of having 'adequate support' and the 'suitability of VC' were similar between patients' view and that of professionals.

Professionals across Health Boards and care sectors reported a range of challenges of $\mathrm{VC}$ when delivering healthcare and social care. There were a wide range of reasons to support their preferences. These are divided into three subcategories and displayed as narrative quotes.

\section{Professional difficulties}

Some professionals report VC to be challenging for some services to carry out their typical work in specialties that required more 'hands-on' healthcare delivery, such as Physiotherapy:

It can be difficult with my profession as a physio as assessments require handling. (Paediatrics Physiotherapist, Secondary care)
A lot of physio input requires physical presence. (Physiotherapist, Secondary care)

Furthermore, professionals experienced eventualities where VC did not work as intended and were required to revert back to other forms of consultation:

We used it initially, but found that picture quality (over and above a good photograph) meant that it added little if anything to a telephone consult. (GP, Primary Care)

\section{Patient difficulties}

Overall, professionals reported more perceived difficulties for their patients than they did for themselves. However, they noted that difficulties for patients were generally situation-specific (eg, where in Wales they live, that is, rural/urban) and depended on each patient's own abilities and experience (eg, using technology):

Some of my patients live in rural areas with poor internet signal, or they simply do not have a computer or phone to access virtual meetings. (Psychiatry Nurse, Secondary care)

For most patients it is excellent, older adults and those without English as a first language find it harder. (Doctor, Primary care)

Working with a very elderly demographic, many do not know how to use tablets, smartphones or PC, [they] prefer telephone. (Domiciliary Optometrist, Primary care)

Despite some of these reported difficulties, many professionals noted that their patients had started to overcome the initial problems by equipping themselves with required resources and improved skills, thus embracing the change to digital care:

Patients are getting used to them. Surprising how even older people's IT skills have up-scaled during the pandemic. (GP, Primary care)

[Despite] unpredictable internet speeds...patients have managed to follow the instructions and engagement with the process has been positive. (Physiotherapist, Secondary care)

\section{Improvements needed}

Many professionals across all Health Boards reported much-needed improvements with access to better and more up-to-date technology, improved connectivity and greater levels of accessibility to produce better VC uptake, outcomes and benefits:

Lack of equipment and internet provided can cause great stress ... there is no WIFI and limited internet ports so not everyone can have a PC at the same time. (Psychologist, Secondary care)

Often depends upon the IT set up of the patient and family. If they have good IT equipment and connection it can work well. (Dietician, Secondary care) 


\section{DISCUSSION}

Throughout the study, the majority of responses were positive, and professionals expressed optimistic views regarding the use, value and benefit of VC, even when faced with challenges on occasions. While responses do for the most part remain positive, a more nuanced approach is important when considering the small and important differences for why a number of responses may be less positive. These differences are highlighted within the challenges and aid in not oversimplifying the study. By using the qualitative aspects of the cross-sectional study, this has led to a more nuanced understanding of $\mathrm{VC}$ across specialities within the NHS.

These data do contribute to the growing evidence for $\mathrm{VC}$ and its benefits and similarities to face-to-face consultations. ${ }^{1-3}$

\section{Use of VC}

Overall, findings suggest that VC should be offered and used where it is deemed clinically appropriate by professionals. The narrative data available support this in suggesting that VC should be used in combination and in addition to face-to-face consultations. A number of professionals did view VC more negatively, predominately associated with holding perceptions of $\mathrm{VC}$, in that it does not provide any further benefits than telephone calls can provide, or as a result of difficulties with accessing technology needed for VC. Similar responses were reported within Primary and Secondary Care, where the majority would consider using $\mathrm{VC}$ where clinically appropriate, with a very small number of professionals suggesting they would not use VC. Despite professionals across Community Care, Management and Administration reporting more negatively on this, the majority suggested that VC should still be offered and used within healthcare.

Regular use of VC was reported by the majority of professionals, and that their colleagues are also keen to use it paired with the adequate training they had received for VC. It is, however, important to note the fairly large numbers of negative comments with respect to equipment, appropriate room availability and sufficient internet connectivity. These comments suggest that professionals require more support in this area which would then allow for VC to be used in a secure and safe manner.

As the narrative suggests, a number of professionals did give lower ratings for $\mathrm{VC}$ being the equivalent to telephone and for VC being the equivalent to face-to-face. This suggests that despite $\mathrm{VC}$ being implemented across many services and specialities in Wales, VC is still not perceived to be the same as in-person face-to-face or even telephone consultations for a number of professionals. While this is evident within the findings, it is important to consider that perhaps it is a reflection of those professionals who view $\mathrm{VC}$ as a temporary measure in the current climate. Regardless of this, it is recommended that VC should be a tool that professionals can use where appropriate, rather than a replacement for either face-to-face or telephone.
What is interesting from the findings is the notable differences between Primary and Secondary Care on the 'true and false' statements. Secondary Care did have a higher proportion of positive responses, including using VC more regularly than Primary Care, having colleagues more eager to use VC and their systems set up for the use of VC. Despite this, Primary Care were more positive in having adequate support, equipment and sufficient internet connectivity. This implies that Secondary Care perceive the need for $\mathrm{VC}$ more, yet lack the resources to do so, compared with colleagues in Primary Care who tend to have the resources, yet are less inclined use it.

\section{Benefits of VC}

On a whole, VC has been perceived as beneficial across all aspects, except for being slightly lower for aspects such as reducing stress and anxiety for their patients and reducing the likelihood of 'DNAs'. However, these were still perceived to be beneficial by professionals, overall (approximately half of the respondents). This is in line with qualitative data, which highlight a large proportion of professionals reporting a reduction in DNAs, with some stating they have had no DNAs since the implementation of VC. While this is an obvious benefit, it is more nuanced than at surface level, and due to COVID-19 restrictions and implications, needs to be interpreted with care. The most positively rated benefit was saving time, travel and parking for both patient and professional. The narrative explores this further considering environmental benefits and revealed that saving travel time for the patient and professional was useful, particularly for those who exhibit difficulties with leaving their homes due to psychological, neurological and physical disorders. Furthermore, these data also suggest that VC makes appointments more accessible for many patients, specifically those with disorders such as autism-spectrum disorders, and for younger patients by allowing them to take a more independent role in their care. However, further research is required to explore this finding. The use of $\mathrm{VC}$ also had benefits for professionals, improving work/life balance, increasing flexibility, and improving communication within and between different teams.

\section{Challenges of VC}

Challenges of VC were perceived to be more relevant for patients than professionals. Differences were identified between professionals' challenges and their perceptions of patient challenges for aspects such as having access to a technology device, internet accessibility and connection, plus an important factor of a lack in confidence. Preference for a face-to-face consultation was experienced as a challenge for professionals and patients, one which does need to be explored and addressed further. By doing so, this will help to support the previously mentioned suggestion that face-to-face and VC are enabled side by side, enabling professionals and patients to make the choice of when face-to-face is appropriate based on clinical judgement and specific patient situations and experiences. 
A section of the qualitative data revealed difficulties with technology, which forms a part of the restraints patients and professionals have within their VC experience. One factor that was seen to impact subjective difficulties was the demographics of patients, particularly older or elderly individuals, who may not be as 'tech-savvy' as the younger generation. Nevertheless, the benefits of VC do seem to outweigh the difficulties, and further support is evidently needed to aid the adjustment to digital healthcare and long-term change of implementation of VC.

\section{Limitations}

Sample bias as the questionnaire was only completed by professionals using the video platforms, and it is difficult to capture the opinion of those who did not use VC. Also, the time period was restricted to only 1 month and we are now capturing prospective data over a longer period using a mixed methodology study as we are keen to understand how we embed our COVID-19 learning into future healthcare and social care delivery. The methodology was restricted to use of questionnaires as face-to-face contact was prohibited, but this ensured a high degree of anonymity for participants and allowed for wider participation of professional staff within NHS Wales, giving voice to a range of perspectives that may have been lost in online focus groups or online interviews, where existing relationships and power structures may have influenced people's responses.

\section{CONCLUSIONS AND RECOMIMENDATIONS}

In response to the emergency pandemic, TEC Cymru implemented an NHS Wales VC Service to all Health Boards in Wales using a single VC platform. This Welsh implementation has approached $\mathrm{VC}$ as a venture requiring co-ordination and collaboration rather than direction, target-driven and traditional methodologies reflecting an emerging trend in improved systems management. ${ }^{20} 21$ TEC Cymru had already established a network through the technical (Assistant Directors of Informatics) and clinical leads (Chief Clinical Information Officers) in Wales, enabling Health Boards to be 'ready to receive' the VC implementation. Building on their existing relationships, an agreed approach to implementation was adopted, involving local organisations making their own decisions about suitable services to engage and to use their local knowledge and networks to determine a process and agreeing standards.

This study also has potential for possible future research. The centralised coordination via TEC Cymru, responsible for providing a framework for planning, resourcing, guidance, training, technical readiness and implementation allied to devolved local clinical and programme teams, could be evaluated separately to establish and provide evidence that this implementation approach and the strategies used were effective, particularly to investigate whether there has been success in maintaining interest and continued use of $\mathrm{VC}$ uptake, knowledge and expansion.

The evaluation of the NHS Wales VC Service (all TEC Cymru data) demonstrates consistency of satisfaction and acceptability across all Health Boards and care sectors, and therefore, a lesson learnt in Wales is that difference is not necessarily negative, but rather a more ideal and suited approach to take when implementing new innovations into the NHS, such as VC to encourage its longterm sustainability. Not only this, but it also highlights that there is no single model that fits all, even in a small country such as Wales.

For an in-depth view of the full report or other data sets captured by TEC Cymru on VC, please visit our website. 223

Twitter Alka Ahuja @Alka Sashin

Acknowledgements Confirmation that the data from Digital Health Wales mentioned within this manuscript have been written by the authors of this paper and therefore have permission to republish.

Contributors GJ contributed to the main design of the study and development of the research questions, the main structure and write-up of the paper, and final amendments to the manuscript. JW and BW analysed the survey data, with GJ and $A A$ involved in supervision. GJ, $A A, S K, J W$ and BW discussed the data once analysed and interpreted the data. SK, MO, KP, MH and AW helped structure the manuscript and contributed to the Programme and clinical understanding of the findings and shaped the conclusions and recommendations. AA was responsible for overseeing the entire development of the study and paper from a clinical and programme perspective, helped structure the manuscript, and shape final conclusions and recommendations. All authors contributed to proof-reading and amendments of the final manuscript. Guarantor: GJ.

Funding TEC Cymru and the National Video Consultation (VC) Service is a funded programme by the Welsh Government (no award number provided).

Competing interests None declared.

Patient consent for publication Not applicable.

Ethics approval TEC Cymru uses a Quality Improvement (QI) methodology to evaluate NHS Wales services, in that typically no ethical approval is required. However, TEC Cymru did obtain full ethical approval and risk assessments from Aneurin Bevan University Health Board Research \& Development combined ethics and risk committee (R\&D Reference Number: SA/1114/20).

Provenance and peer review Not commissioned; externally peer reviewed.

Data availability statement Data are available upon reasonable request. All data relevant to the study are included in the article or uploaded as supplementary information. Data made available upon reasonable request.

Supplemental material This content has been supplied by the author(s). It has not been vetted by BMJ Publishing Group Limited (BMJ) and may not have been peer-reviewed. Any opinions or recommendations discussed are solely those of the author(s) and are not endorsed by BMJ. BMJ disclaims all liability and responsibility arising from any reliance placed on the content. Where the content includes any translated material, BMJ does not warrant the accuracy and reliability of the translations (including but not limited to local regulations, clinical guidelines, terminology, drug names and drug dosages), and is not responsible for any error and/or omissions arising from translation and adaptation or otherwise.

Open access This is an open access article distributed in accordance with the Creative Commons Attribution Non Commercial (CC BY-NC 4.0) license, which permits others to distribute, remix, adapt, build upon this work noncommercially, and license their derivative works on different terms, provided the original work is properly cited, appropriate credit is given, any changes made indicated, and the use is non-commercial. See: http://creativecommons.org/ licenses/by-nc/4.0/.

ORCID iD

Gemma Johns http://orcid.org/0000-0001-9823-4822 


\section{REFERENCES}

1 Wherton J, Shaw S, Papoutsi C, et al. Guidance on the introduction and use of video consultations during COVID-19: important lessons from qualitative research. BMJ Leader 2020;4:120-3.

2 Greenhalgh T, Wherton J, Shaw S, et al. Video consultations for covid-19. BMJ 2020;368:m998.

3 Johns G, Tan J, Burhouse A, et al. A visual step-by-step guide for clinicians to use video consultations in mental health services: NHS examples of real-time practice in times of normal and pandemic healthcare delivery. BJPsych Bull 2020;44:277-84.

4 Donaghy E, Atherton H, Hammersley V, et al. Acceptability, benefits, and challenges of video consulting: a qualitative study in primary care. Br J Gen Pract 2019;69:e586-94.

5 Darnton R, Lopez T, Anil M, et al. Medical students consulting from home: a qualitative evaluation of a tool for maintaining student exposure to patients during lockdown. Med Teach 2021;43:1-8.

6 Connor MJ, Winkler M, Miah S. COVID-19 pandemic - is virtual urology clinic the answer to keeping the cancer pathway moving? BJU Int 2020;125:E3-4.

7 Jones B, Scott J. Building The Evidence Base On Video Consultations | The Health Foundation. [online] The Health Foundation, 2020. Available: https://www.health.org.uk/news-andcomment/blogs/building-the-evidence-base-on-video-consultations

8 Greenhalgh T, Shaw S, Wherton J, et al. Real-World implementation of video outpatient consultations at macro, meso, and micro levels: mixed-method study. J Med Internet Res 2018;20:e150.

9 Marshall M, Shah R, Stokes-Lampard H. Online consulting in genera practice: making the move from disruptive innovation to mainstream service. BMJ 2018;360:k1195.

10 Cresswell K, Sheikh A. Organizational issues in the implementation and adoption of health information technology innovations: an interpretative review. Int J Med Inform 2013;82:e73-86.

11 et alSheikh A, Cornford T, Barber N. Implementation and adoption of nationwide electronic health records in secondary care in England: final qualitative results from prospective. Available: https://linkinghub. elsevier.com/retrieve/pii/S1386505612001992

12 Wales Audit Office. Informatics systems in NHS Wales informatics systems in NHS Wales Archwilydd Cyffredinol Cymru auditor General for Wales, 2018.

13 National Assembly for Wales Public Accounts Committee. Informatics systems in NHS Wales, 2018. Available: https://www. audit.wales/system/files/publications/NHS_-Informatics-2018 English.pdf

14 Technology enabled care (Tec) Cymru. Available: https://digitalhealth wales/tec-cymru

15 Wachter RM. Making IT Work: Harnessing the Power of Health Information Technology to Improve Care in England [Internet], 2016. Available: https://assets.publishing.service.gov.uk/government/ uploads/system/uploads/attachment_data/file/550866/Wachter_ Review_Accessible.pdf [Accessed 08 May 2019].

16 Agile delivery - Service Manual - GOV.UK [Internet]. Available: https:// www.gov.uk/service-manual/agile-delivery [Accessed 11 Dec 2020].

17 Greenway A, Terrett B, Bracken M. Digital transformation at scale: why the strategy is delivery. first. London: London Publishing Partnership, 2018.

18 Vize R. The revolution will be improvised stories and insights about transforming systems, 2016.

19 Mintzberg H. Managing the myths of health care. first. Berret-Koehler Publishers, Inc: Oakland, 2017.

20 Johns G, Khalil S, Ogonovsky M, et al. Video consulting contributes to carbon neutral healthcare. BMJ 2020;371:m4300.

21 The NHS Wales video consulting service, 2020. Available: https:// digitalhealth.wales/tec-cymru/vc-service

22 Technology enabled care (Tec) Cymru - the professional perspective report. Available: https://digitalhealth.wales/sites/default/files/202012/P1\%20Report\%20-\%20Chapter\%202\%20Retrospective\% 20Data_V1.0_Dec2020.pdf

23 Technology enabled care (Tec) Cymru - the evidence. Available: https://digitalhealth.wales/tec-cymru/how-we-can-help/evidence 